\title{
Clustering in a Precipitate-Free GeMn Magnetic Semiconductor
}

\author{
D. Bougeard, ${ }^{1}$ S. Ahlers, ${ }^{1}$ A. Trampert, ${ }^{2}$ N. Sircar, ${ }^{1}$ and G. Abstreiter ${ }^{1}$ \\ ${ }^{1}$ Walter Schottky Institut, Technische Universität München, Am Coulombwall 3, D-85748 Garching, Germany \\ ${ }^{2}$ Paul-Drude-Institut für Festkörperelektronik, Hausvogteiplatz 5-7, D-10117 Berlin, Germany
}

(Received 19 May 2006; published 7 December 2006)

\begin{abstract}
We present the first study relating structural parameters of precipitate-free $\mathrm{Ge}_{0.95} \mathrm{Mn}_{0.05}$ films to magnetization data. Nanometer-sized clusters - areas with increased Mn content on substitutional lattice sites compared to the host matrix - are detected in transmission electron microscopy analysis. The films show no overall spontaneous magnetization at all down to $2 \mathrm{~K}$. The TEM and magnetization results are interpreted in terms of an assembly of superparamagnetic moments developing in the dense distribution of clusters. Each cluster individually turns ferromagnetic below an ordering temperature which depends on its volume and Mn content.
\end{abstract}

PACS numbers: 75.50.Pp, 68.37.Lp, 75.75.+a, 81.15.Hi

The development of a novel class of materials combining standard semiconductors with magnetic elements has recently been driven by considerable technological as well as fundamental scientific interest. While the possibility of a seamless combination of magnetic and semiconducting systems using spins as an additional degree of freedom opens stimulating perspectives in the field of electronics $[1,2]$, reports on materials displaying both semiconducting and ferromagnetic properties have induced great theoretical and experimental efforts in the understanding of the underlying physics [3]. $\mathrm{Ga}(\mathrm{Mn})$ As today represents one of the best understood ferromagnets. This material is one example of a diluted magnetic semiconductor (DMS), meaning a dispersion of the magnetic elements without affecting the semiconducting properties of the matrix [4]. The realization of DMS with maximized ferromagnetic ordering temperatures $T_{C}$ represents the ultimate objective in this field.

Special attention has been given to technologically important group IV semiconductor based magnetic materials, with a prominent position for GeMn. Since the first claim of the realization of a Ge based DMS [5], most publications [5-8] have concentrated on reporting high $T_{C}$ ranging from 116 [5] to $285 \mathrm{~K}$ [6] and on interpreting the observed ferromagnetism in terms of DMS theories [9]. It is only recently that several of the former GeMn reports have been questioned by structural proofs [10] and hints [11] for the formation of intermetallic ferromagnetic compounds through phase separation in single crystals and lowtemperature molecular beam epitaxy (MBE) fabricated films, respectively. Up to now, only Li et al. [11] presentindirect - indications for the realization of precipitate-free GeMn. Considering the current discussion on the magnetic properties of GeMn, a study of the crystal structure, exploring the degree of $\mathrm{Mn}$ dispersion that can be reached in $\mathrm{Ge}$, would obviously be beneficial for the field.

In this Letter, we present the first study relating structural parameters of precipitate-free $\mathrm{Ge}_{0.95} \mathrm{Mn}_{0.05}$ films to magnetization data, providing new insights into the inter- pretation of the magnetic properties of GeMn. Although the incorporation of $\mathrm{Mn}$ does not induce explicit phase separation, nanometer-sized areas with increased Mn content compared to the surrounding matrix are detected in transmission electron microscopy (TEM) analysis. The films show no overall spontaneous magnetization down to $2 \mathrm{~K}$. The comparison of the TEM analysis and magnetization data indicates the formation of magnetic supermoments due to an inhomogeneous dispersion of $\mathrm{Mn}$ in the $\mathrm{Ge}$ host matrix. Supermoments appear below a characteristic ordering temperature, which depends on the volume and the Mn content of one particular area.

The samples were produced in low-temperature MBE on intrinsic $\mathrm{Ge}(001)$ substrates. After a thermal desorption of the natural oxide, a flat Ge buffer layer was deposited at a substrate temperature $T_{S}$ of $280^{\circ} \mathrm{C}$. A careful variation of the substrate temperature during the subsequent codeposition of nominally $95 \% \mathrm{Ge}$ and $5 \% \mathrm{Mn}$ has shown that a film which is free of intermetallic precipitates was only reproducibly obtained with $T_{S}$ as low as $60^{\circ} \mathrm{C}$ and with a Ge deposition rate of $0.08 \AA / \mathrm{s}$. The details of this study will be published elsewhere [12]. The typical film thickness is $200 \mathrm{~nm}$. Structural analysis of the films was performed through x-ray diffraction (XRD) and extensive TEM studies on a JEOL 3010 microscope operating at $300 \mathrm{kV}$. Special care was taken during sample preparation to avoid unintentional postgrowth annealing. Chemical analysis was obtained by electron energy loss spectroscopy (EELS). A commercial superconducting quantum interference device (SQUID) was used for magnetization measurements.

No intermetallic precipitates could be detected in XRD for samples with $T_{S}=60^{\circ} \mathrm{C}$. Conventional TEM observations on cross-sectional samples (not shown) display perfect epitaxy of the $\mathrm{Ge}_{0.95} \mathrm{Mn}_{0.05}$ film on the Ge buffer layer. The cubic crystalline structure of the buffer layer is conserved. No dislocation was observed in several TEM micrographs covering few microns. Furthermore, the absence of phase separation indicates a film free of interme- 


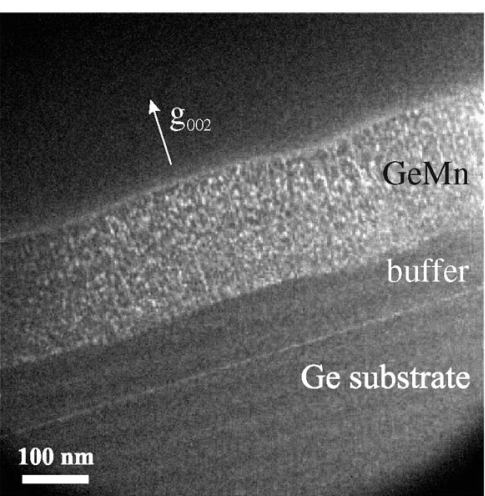

FIG. 1. Dark field TEM overview of a $\mathrm{Ge}_{0.95} \mathrm{Mn}_{0.05}$ film fabricated in $\mathrm{MBE}$ at $T_{S}=60^{\circ} \mathrm{C}$. Contrast is obtained by the selection of the chemically sensitive (002) Ge reflex. Bright areas correspond to an enhanced Mn content.

tallic precipitates. Nevertheless, by selecting the chemically sensitive (002) Ge reflex in dark field microscopy mode, as depicted in Fig. 1, a dense distribution of almost round shaped, nanometer-sized areas appeared in the whole $\mathrm{Ge}_{0.95} \mathrm{Mn}_{0.05}$ film. These bright spots represent areas with increased Mn concentration on substitutional sites of the lattice compared to the darker matrix, since the (002) reflection is forbidden in the diamond cubic structure of Ge. The areas will be denoted as clusters in the following. Their typical diameter is $4 \mathrm{~nm}$. EELS measurements with $100 \mathrm{~nm}$ probe size confirm the stoichiometry of the $\mathrm{Ge}_{0.95} \mathrm{Mn}_{0.05}$ alloy for the film. A comparative EELS analysis of the clusters and the matrix is hampered by their very small size and dense distribution. An upper limit of $15 \%$ for the average Mn content on substitutional sites in the clusters is estimated through the ratio of dark and bright areas supposing that all of the $\mathrm{Mn}$ atoms are incorporated into the clusters. Different levels of brightness of the clusters in the dark field analysis indicate a slight variation in the Mn content from cluster to cluster. The observations in dark field TEM are confirmed by high-resolution TEM micrographs. A typical example is shown in Fig. 2. The lattice image reveals areas with slightly darker contrast but still reflecting the same lattice symmetry; that is, these areas are coherently bound to the surrounding. They are identified to the clusters shown in Fig. 1 as they have the same shape and dimensions. Thus, the GeMn layer, while being free of intermetallic precipitates, is composed of a dense assembly of well-defined, coherent, nanometer-sized areas showing either high or low Mn content.

Temperature-dependent magnetization measurements for different external magnetic fields are shown in Fig. 3. For all curves, the sample was cooled down in the maximum available field of $\mu_{0} H=7 \mathrm{~T}$ (maximum field cooled, MFC) and the data recorded during warm-up in the measurement field. If no external field was applied during the measurement, the magnetization decreased from a finite value at $2 \mathrm{~K}$ to zero at about $18 \mathrm{~K}$. No magnetization at all was detected above this temperature.

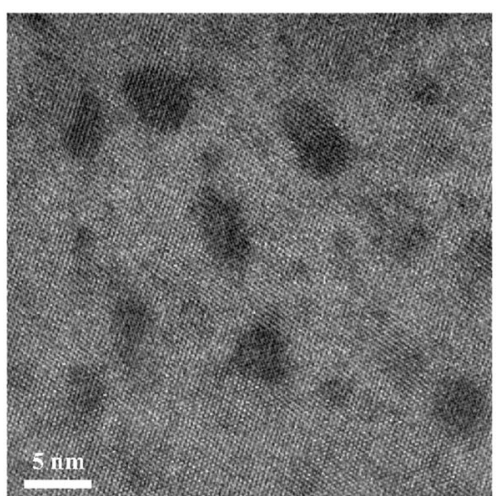

FIG. 2. Typical high-resolution TEM micrograph of $\mathrm{Ge}_{0.95} \mathrm{Mn}_{0.05}$. Dark contrast reveals cubic clusters which are coherently bound to the surrounding.

Nonzero external magnetic fields in contrast induced nonvanishing magnetization values from approximately 18 up to approximately $200 \mathrm{~K}$ while conserving the steep decrease between 2 and $18 \mathrm{~K}$. The magnetic properties of the GeMn layer thus seem to be characterized by two distinct domains separated at a critical temperature of about $18 \mathrm{~K}$. Above this temperature, an overall spontaneous magnetization of the film can be excluded.

Field-dependent magnetization loops recorded below and above $18 \mathrm{~K}$ are shown in Fig. 4. Again the sample was cooled down to the measurement temperature in MFC conditions for all of the loops. A hysteresis is observed for $6.5 \mathrm{~K}$, as displayed in the upper inset, with a remanent magnetization of $0.73 \mathrm{kA} / \mathrm{m}$ and a coercive field of $\mu_{0} H_{c}=41 \mathrm{mT}$. The hysteresis gradually vanishes with increasing measurement temperature towards approximately $15 \mathrm{~K}$. The loops for higher temperatures are then reminiscent of an atomic paramagnet. But neither does the high-field saturation of the loops vanish towards higher temperatures as expected for atomic paramagnets, nor can the loops be approximated by Brillouin curves using reasonable $g$ and $J$ parameters. They can, in turn, be described in the infinite limit of the Brillouin function, that is, by a

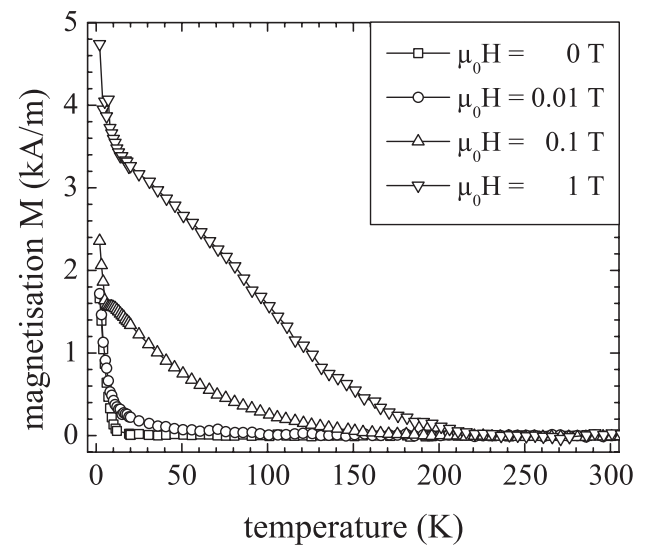

FIG. 3. Temperature dependence of the MFC magnetization of $\mathrm{Ge}_{0.95} \mathrm{Mn}_{0.05}$ for external fields between 0 and $1 \mathrm{~T}$. 


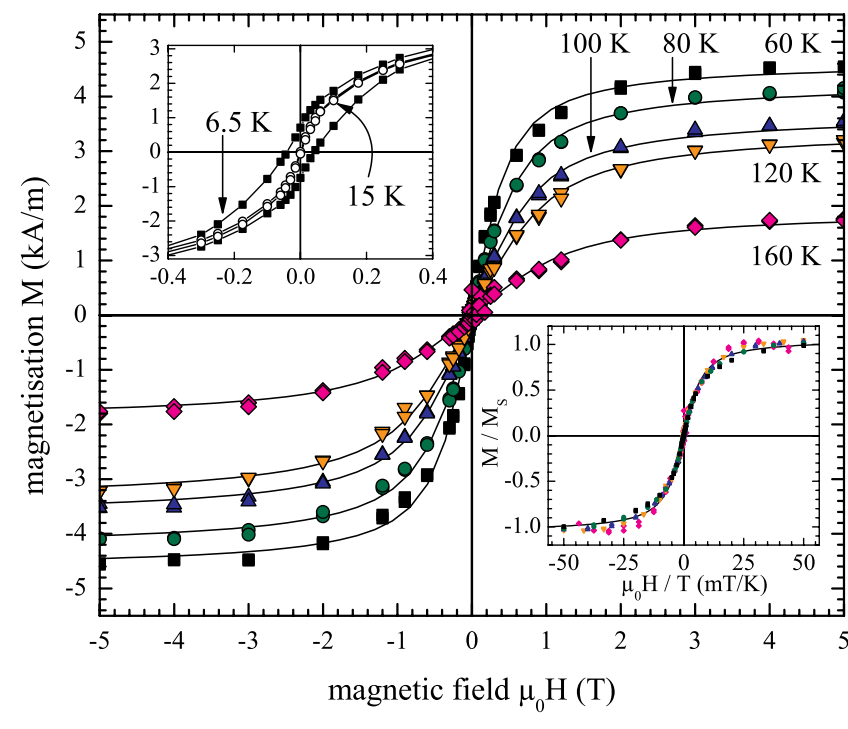

FIG. 4 (color online). Magnetization loops taken between 60 and $160 \mathrm{~K}$. Solid lines represent fits with the Langevin function with $\mu=435 \mu_{B}$. (Upper inset) Magnetization loops at 6.5 and $15 \mathrm{~K}$. (Lower inset) $M / M_{S}$ versus $H / T$. The symbols represent the same temperature series as in the main figure. The solid line is a Langevin fit with $\mu=435 \mu_{B}$.

Langevin behavior, as indicated by the solid lines in Fig. 4, with

$$
M(y)=M_{\mathrm{S}}\left(\operatorname{coth} y-\frac{1}{y}\right), \quad y=\frac{\mu \mu_{0} H}{k_{B} T},
$$

where $M$ represents the magnetization, $M_{S}$ the saturation magnetization of the cluster ensemble, and $\mu$ the average magnetic moment per cluster. For each measurement temperature, the best Langevin fit is obtained for $\mu=435 \mu_{B}$. Moreover, when the same data sets for $T=60-160 \mathrm{~K}$ are plotted as $M$ over $M_{S}$ versus external field $H$ over temperature $T$, the data points superimpose as shown in the lower inset in Fig. 4. The Langevin fit of this plot again gives $\mu=435 \mu_{B}$. This behavior is characteristic for superparamagnetism [13].

Typical clusters have a diameter of 3-4 nm and 10\%$15 \%$ of Mn atoms incorporated on substitutional sites, as identified in TEM. Assuming a spherical shape and an average moment per $\mathrm{Mn}$ atom of $1-3 \mu_{B}[5,11,14]$, we evaluate a magnetic moment of about $100-600 \mu_{B}$ per cluster. The order of magnitude of this estimation is in good agreement with the average magnetic moment of $\mu=435 \mu_{B}$ extracted from the Langevin fits in Fig. 4, while it is much smaller than the typical moment obtained for intermetallic precipitates for $T_{\mathrm{S}}>60 \mathrm{~K}$ [12]. The comparison of the structural and magnetic properties above $20 \mathrm{~K}$ thus indicates that the magnetic moments responsible for the superparamagnetism are located in the clusters shown in Fig. 1. An individual supermoment is then created when a single cluster turns ferromagnetic below a certain Curie temperature $T_{C}^{\text {Cluster }}$.
The high-field saturation magnetization $M_{S}$ of the magnetization loops in Fig. 4 decreases when the measurement temperature is increased. Since at the same time the average magnetic moments extracted from the Langevin fits stay at a constant level for different measurement temperatures, fewer clusters seem to contribute to $M_{S}$. This is equivalent to an increasing number of clusters that cross their individual $T_{C}^{\text {Cluster }}$ and therefore lose their ferromagnetic order. Thus, magnetometry as well as the different Mn contents $[3,11]$ observed in TEM indicate the presence of a distribution of $T_{C}^{\text {Cluster }}$ in the cluster ensemble.

As shown in the lower inset in Fig. 4, the system is superparamagnetic up to at least $160 \mathrm{~K}$, which represents a lower limit for the maximum value of $T_{C}^{\text {Cluster }}$. An upper limit for this maximum cannot be extracted from these data, mainly due to a low signal level above $160 \mathrm{~K}$. The presence of a nonzero magnetization in $\mu_{0} H=1 \mathrm{~T}$ at $200 \mathrm{~K}$ further suggests that the maximum achievable $T_{C}^{\text {Cluster }}$ is larger than $160 \mathrm{~K}$. In preliminary magnetic characterization, the curve form of postgrowth annealed films as well as of ones with Mn content up to $8 \%$ do not differ from Figs. 3 and 4. The obvious difference is a shift of the onset of magnetization in nonzero field to values larger than $200 \mathrm{~K}$. This is consistent with an expected shift of the distribution of supermoments and $T_{C}^{\text {Cluster }}$ to higher values when increasing the $\mathrm{Mn}$ concentration.

Figures 3 and 4 at first glance seem to indicate the presence of a spontaneous magnetization below a critical temperature of approximately $18 \mathrm{~K}$. However, the magnetic properties below $18 \mathrm{~K}$ depend on the sample history. This can be observed in relaxation measurements for zero field cooled (ZFC) samples in Fig. 5(a), where magnetization is measured versus time after switch-off of an externally applied field. There is an obvious relaxation of the magnetization for temperatures below $15 \mathrm{~K}$. Above $15 \mathrm{~K}$, relaxation is strongly suppressed. The solid lines in Fig. 5(a) represent fits to a stretched exponential decay $\sim \exp \left[-(t / \tau)^{1-n}\right]$, with $n$ values between 0.5 and 0.6. In Fig. 5(b), a peak in the real part of the low-field ac susceptibility measured at different measurement frequencies is observed at temperatures around $14 \mathrm{~K}$. This susceptibility peak changes slightly in both height and position depending on the measurement frequency. The relative shift of the peak position per frequency decade is 0.03 . We interpret the measurements in terms of a metastable state. This metastable state can be induced by a multivalley structure in the free energy phase space of the system at low temperatures, resulting in magnetization relaxation phenomena that become visible in aging effects and in a peak shift of the ac susceptibility [15]. Further indications supporting this interpretation are the observation of a nonreversibility of magnetization at $18 \mathrm{~K}$ in Fig. 5(c), that is, a bifurcation of field cooled (FC) and ZFC temperaturedependent magnetization measurements, and a peak at $18 \mathrm{~K}$ in the ZFC curves. Furthermore, measurements taken during zero field cooldown lead to a zero magnetic signal 


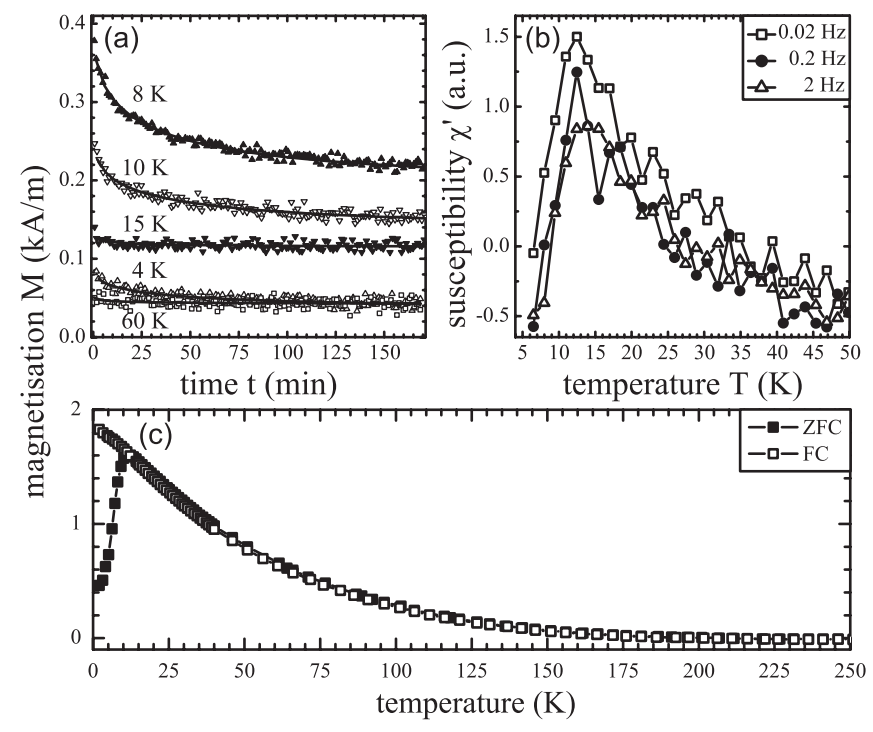

FIG. 5. (a) Relaxation of the magnetization of $\mathrm{Ge}_{0.95} \mathrm{Mn}_{0.05}$ after switching off the external field at different temperatures. Solid lines represent fits to a stretched exponential decay. (b) Temperature-dependent real part of ac susceptibility measured at different frequencies. (c) FC or ZFC temperaturedependent magnetization. $\mu_{0} H=0.1 \mathrm{~T}$.

(not shown). A multivalley structure of the free energy landscape below $15 \mathrm{~K}$ can be due to the existence of a glasslike state or interacting blocked particles [16]. Neither of the two possibilities can be excluded from our measurements. Both of them would induce the steep decrease in magnetization between 2 and $18 \mathrm{~K}$ in Fig. 3 as well as the gradually vanishing hysteresis in Fig. 4 in the same temperature range. Nevertheless, the clear indications for metastability make a transition to a ferromagnetic state highly unlikely. Furthermore, temperature-dependent resistivity measurements of our samples reveal typical insulating behavior with a diverging resistivity towards zero temperature. Irregularities in the temperature-dependent resistivity curve are expected in the case of magnetic ordering $[11,17,18]$. No irregularity and, therefore, no hint for a magnetic percolation are observed in the temperature range where the system undergoes a transition between the superparamagnetic to a metastable regime in our samples. Thus, from our experiments, suggesting welldefined and stable clusters with enhanced Mn concentration on substitutional Ge lattice sites, we cannot confirm a ferromagnetic percolation [11] conjectured to result from magnetic "spin clusters" which grow in size with decreasing temperature. We suggest that the ferromagnetism of a cluster is mediated by holes which are spatially localized inside this cluster [17,19].

To conclude, we have shown that as-grown $\mathrm{Ge}_{0.95} \mathrm{Mn}_{0.05}$ layers free of intermetallic precipitates produced by lowtemperature $\mathrm{MBE}$ at $T_{S}=60^{\circ} \mathrm{C}$ are very close to the $\mathrm{Mn}$ dispersion level which can be realistically expected for this kind of extreme epitaxy conditions. We report the first observation of Mn enriched clusters with an upper limit of $15 \% \mathrm{Mn}$ per cluster. The magnetic properties of GeMn films are clearly a consequence of the dense ensemble of nanometer-sized clusters. Each of these clusters turns ferromagnetic below its own characteristic transition temperature $T_{C}^{\text {Cluster }}$, which depends on its Mn content and its volume. The ferromagnetism of a cluster is mediated through holes which can freely move only inside this cluster. The surrounding matrix in contrast seems to lack freely moving holes. The clusters then carry individual magnetic supermoments which lead to the observation of superparamagnetism above approximately $18 \mathrm{~K}$ and a metastable state at lower temperatures. The physical origins of the metastable state have to be further investigated. The lower limit of the maximal $T_{C}^{\text {Cluster }}=160 \mathrm{~K}$ confirms the potential of a Ge based DMS for spintronic applications.

This work was supported by Deutsche Forschungsgemeinschaft via SFB 631. The authors gratefully acknowledge access to the SQUID magnetometer at the Walther-Meissner-Institut, Garching, as well as discussions with R. Gross, M. Opel, C. Jäger, C. Bihler, and M. S. Brandt.

Note added.-Recently, we learned of a related work [20] reporting the observation of GeMn nanocolumns.

[1] S. A. Wolf, A. Y. Chtchelkanova, and D. M. Treger, IBM J. Res. Dev. 50, 101 (2006).

[2] M. Tanaka, J. Cryst. Growth 278, 25 (2005).

[3] T. Dietl et al., Science 287, 1019 (2000).

[4] H. Ohno, Science 281, 951 (1998).

[5] Y. D. Park et al., Science 295, 651 (2002).

[6] S. Cho et al., Phys. Rev. B 66, 033303 (2002).

[7] F. Tsui et al., Phys. Rev. Lett. 91, 177203 (2003).

[8] F. D’Orazio et al., J. Magn. Magn. Mater. 272, 2006 (2004).

[9] N. Pinto et al., Phys. Rev. B 72, 165203 (2005).

[10] J.-S. Kang et al., Phys. Rev. Lett. 94, 147202 (2005).

[11] A. P. Li et al., Phys. Rev. B 72, 195205 (2005).

[12] S. Ahlers et al., Phys. Rev. B (to be published).

[13] I. S. Jacobs and C. P. Bean, in Magnetism, edited by G. T. Rado and H. Suhl (Academic, New York, 1963), Vol. III.

[14] A. Stroppa, S. Picozzi, A. Continenza, and A. J. Freeman, Phys. Rev. B 68, 155203 (2003).

[15] J. A. Mydosh, Spin Glasses: An Experimental Introduction (Taylor \& Francis, London, 1993).

[16] J. L. Dormann, D. Fiorani, and E. Tronc, J. Magn. Magn. Mater. 202, 251 (1999).

[17] A. Kaminski and S. Das Sarma, Phys. Rev. B 68, 235210 (2003).

[18] A. M. Nazmul, S. Sugahara, and M. Tanaka, Phys. Rev. B 67, 241308(R) (2003).

[19] J. Jaroszyński et al., cond-mat/0509189.

[20] M. Jamet et al., Nat. Mater. 5, 653 (2006). 